\title{
SPatio-Temporal VARIAbility of Water VAPOR in The Free Troposphere INVESTIGATED BY DIAL AND FTIR VERTICAL SOUNDINGS
}

\author{
H. Vogelmann, R. Sussmann, T. Trickl, A. Reichert \\ Karlsruhe Institute of Technology, IMK-IFU (Garmisch-Partenkirchen,Germany), \\ E-mail: hannes.vogelmann@kit.edu
}

\begin{abstract}
We report on the free tropospheric spatio-temporal variability of water vapor investigated by the analysis of a five-year period of water vapor vertical soundings above Mt. Zugspitze (2962 m a.s.l., Germany). Our results are obtained from a combination of measurements of vertically integrated water vapor (IWV), recorded with a solar Fourier Transform InfraRed (FTIR) spectrometer and of water vapor profiles recorded with the nearby differential absorption lidar (DIAL). The special geometrical arrangement of one zenith-viewing and one sun-pointing instrument and the temporal resolution of both optical instruments allow for an investigation of the spatiotemporal variability of IWV on a spatial scale of less than one kilometer and on a time scale of less than one hour. We investigated the short-term variability of both IWV and water vapor profiles from statistical analyses. The latter was also examined by case studies with a clear assignment to certain atmospheric processes as local convection or long-range transport. This study is described in great detail in our recent publication [1].
\end{abstract}

\section{INSTRUMENTATION AND GEOGRAPHICAL ARRANGEMENT}

The Zugspitze $\left(47.42^{\circ} \mathrm{N}, 10.98^{\circ} \mathrm{E}, 2962 \mathrm{~m}\right.$ a.s.l.) is by far the highest mountain on the northern rim of the Alps. The free troposphere above this site is representative of Central Europe. The mountain is above the moist boundary layer for most of the year. Due to reduced absorption losses this site is ideal for sensitive spectroscopic measurements of water vapor throughout the free troposphere. While the FTIR instrument is located on the summit of Mt. Zugspitze the DIAL instrument is located at the Schneefernerhaus research station (UFS) on the steep southern slope of Mt. Zugspitze at an altitude of $2675 \mathrm{~m}$ a.s.1., $680 \mathrm{~m}$ southwest of the FTIR instrument (Fig. 1).

The solar FTIR instrument uses direct radiation from the sun in the mid-infrared as light source (Table). The instrument provides water vapor columns integrated along its slanted viewline towards the sun with a precision better than $0.05 \mathrm{~mm}$. It is based on a Bruker IFS125HR interferometer and is described in detail by [2].

The Zugspitze DIAL is operated with single absorption lines in the 817-nm band of $\mathrm{H}_{2} \mathrm{O}$ (see table) for groundbased water vapor profiling in the free troposphere. In order to keep a balanced signal-to-noise ratio a vertical resolution of $50 \mathrm{~m}$ to $300 \mathrm{~m}$ is adapted dynamically to

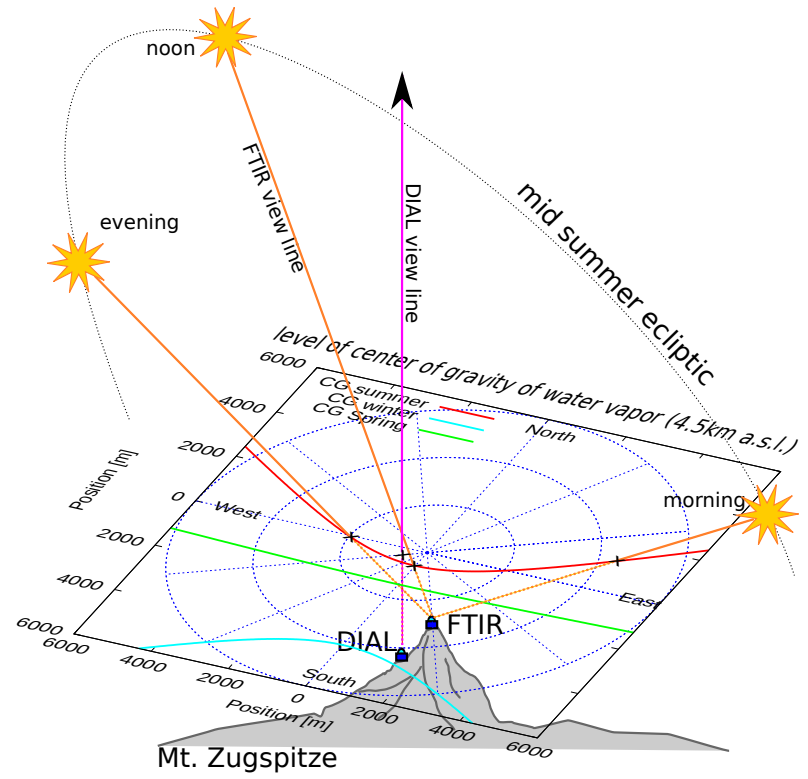

Figure 1: Geographical collocation of the water-vapor DIAL and the FTIR instrument on Mt. Zugspitze

the vertical range from $2.95 \mathrm{~km}$ to roughly $12 \mathrm{~km}$ a.s.l., respectively. Thus, statistical measurement uncertainties are kept below about $5 \%$ related to a mean humidity profile to about $8 \mathrm{~km}$. The sensitivity limit is roughly $18 \mathrm{ppm}$ at $10 \mathrm{~km}$ a.s.1.. The DIAL system at UFS and the retrieval of water vapor profiles are described in more detail by [3]. Water-vapor profiles from the Zugspitze DIAL allow for retrieving IWV with a precision better than $0.1 \mathrm{~mm}$ [4].

\begin{tabular}{l|ll} 
& FTIR & DIAL \\
\hline Geographical & E $10^{0} 59^{\prime} 8.7^{\prime \prime}$ & E $10^{0} 58^{\prime} 46.8^{\prime \prime}$ \\
Coordinates & $\mathrm{N} 47^{0} 25^{\prime} 15.6^{\prime \prime}$ & $\mathrm{N} 47^{0} 25^{\prime} 0^{\prime \prime}$ \\
Altitude a.s.l. & $2964 \mathrm{~m}$ & $2675 \mathrm{~m}$ \\
Range a.s.l. & above $2.96 \mathrm{~km}$ & $2.95 \mathrm{~km}-12 \mathrm{~km}$ \\
Int. time & 13.3 min & $17 \mathrm{~min}$ \\
Spectral & micro windows & $\nu_{\text {on }}$ \\
range $\left[\mathrm{cm}^{-1}\right]$ & $839.5-840.5$ & 12236.560 \\
& $849.0-850.2$ & 12237.466 \\
& $852.0-853.1$ & 12243.537
\end{tabular}

Combining the sun-pointing geometry of the FTIR instrument and the fixed zenith-pointing geometry of the DIAL is ideal for studies of the variations of IWV values measured by both instruments with a defined spatial and temporal matching. For simplicity, it is assumed that 


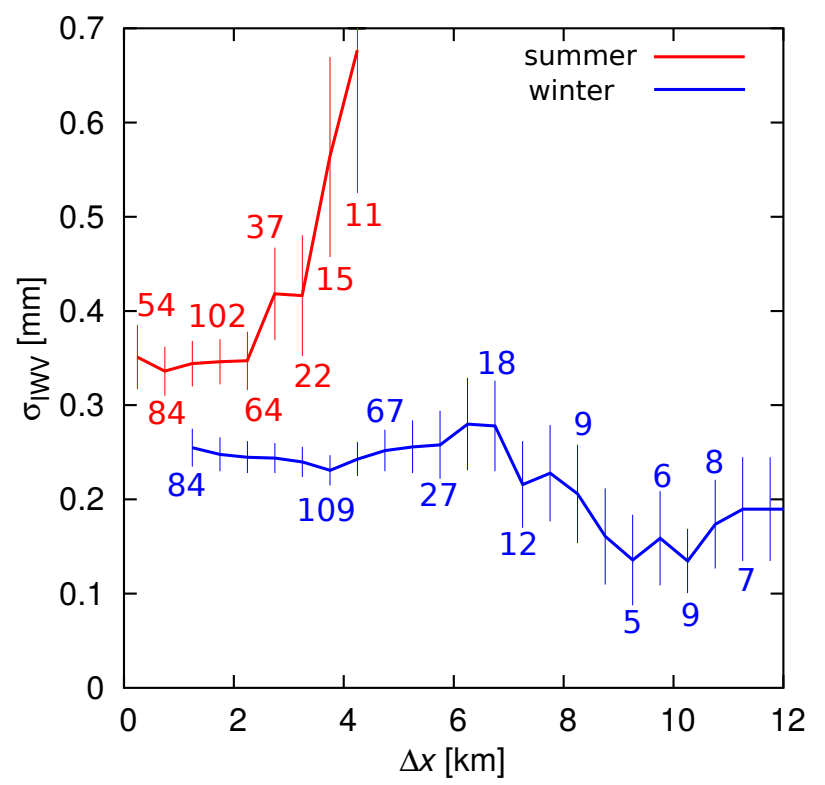

Figure 2: $\sigma_{\mathrm{IWV}}$ as a function of the horizontal distance $\Delta x$ between the center of gravity of FTIR IWV and DIAL IWV in the summer season (red) and in the winter season (blue). The coincidence time interval $\Delta t$ is $60 \mathrm{~min}$ for the blue curve and $30 \mathrm{~min}$ for the red curve. For geometrical reasons, the shortest distance in the winter season is $1 \mathrm{~km}$. The number of measurement pairs from which $\sigma_{\text {IWV }}$ was calculated is indicated by the numbers near the curves (not for all nodes). The uncertainties $( \pm \sigma)$ are indicated by the error bars (for calculation see text).

the FTIR IWV is horizontally located at the point where the viewing direction of the instrument meets the altitude level of the center of gravity of the IWV distribution. From this a rough estimate of the varying horizontal position of the IWV measured by the FTIR instrument is possible. The zenith angle of the sun defines the horizontal distance from the instrument, which may vary from less than $1 \mathrm{~km}$ around noon in midsummer to more than $10 \mathrm{~km}$ at very low sun positions. The azimuth of the FTIR IWV position is equal to the azimuth of the sun position which depends on daytime and season. In contrast to this, the horizontal position of IWV measured with the DIAL is always fixed to the location of the instrument, i.e., $680 \mathrm{~m}$ southwest of the FTIR site.

\section{VARIABILITY OF INTEGRATED WATER VA- POR IN SPACE AND TIME}

Of more than 350 lidar profiles recorded in the years 2007-2009 more than 250 profiles were measured during daytime (i.e. between 5h00 and 19h00 local time). In the same period, more than 3500 column measurements were made by the FTIR instrument. The systems operate with a typical integration time of 13 min (FTIR) and 17 min (DIAL). In order to obtain a quantitative measure of the water vapor variability, we analyzed certain measurement samples recorded by the two different in- struments under certain spatio-temporal matching criteria for $\Delta x$ and $\Delta t$. The center of the integration time of FTIR and DIAL each was used to determine the temporal matching. We retrieved $\sigma_{\text {IWV }}$ by calculating the standard deviation of the differences of IWV values from a linear model $y=a \cdot x+b$ :

$\sigma_{\mathrm{IWV}}=\sqrt{\frac{1}{n-2} \sum_{i=1}^{k}\left(y_{i}-\left(a x_{i}+b\right)\right)^{2}}$,

whereat $y_{i}$ and $x_{i}$ are the IWV-values from the DIAL and the FTIR, respectively, within one sample and $n$ is the sample size. $a$ and $b$ were calculated by a regression analysis using the method of least squares. Thus,

$\sigma_{\mathrm{IWV}}=\sqrt{\frac{1}{n-2} \sum_{i=1}^{n}\left(y_{i}-\bar{y}-\frac{\left(x_{i}-\bar{x}\right) \sum_{j}\left(x_{j}-\bar{x}\right) y_{j}}{\sum_{j}\left(x_{j}-\bar{x}\right)^{2}}\right)^{2}}$.

\section{Spatial variability}

Spatial and temporal variabilities are analyzed separately for summer and winter because of two counteracting effects:

1. The special observation geometry in this study implies that the spatial overlap $\Delta x$ of both soundings depends on both daytime and season. The best spatial matching $(\Delta x<1 \mathrm{~km})$ is achieved around midsummer in the early afternoon only (between $12 \mathrm{~h}$ and $14 \mathrm{~h}$ UTC), while $\Delta x$ is always larger during the winter season.

2. Due to heat-driven convective dynamics in complex mountain surroundings, spatial and temporal variabilities of IWV are expected to be higher during summer season. The convection above alpine terrain can reach an altitude of about $1.5 \mathrm{~km}$ above the mean summit levels in summer. During all other seasons, the convection usually does not even reach the Zugspitze summit and our measurement range.

Figure 2 (red curve) shows $\sigma_{\text {IWV }}$ as a function of the horizontal distance of the probed volumes in the summer season. While $\sigma_{\text {IWV }}$ constantly remains around $0.35 \mathrm{~mm}$ for $\Delta x<2 \mathrm{~km}$, it rises to values of more than $0.65 \mathrm{~m}$ at a distance of $\Delta x=4 \mathrm{~km}$. This result shows that the variability depends on the spatio-temporal matching. Up to $\Delta x=2 \mathrm{~km}$, the temporal variability within the selected time interval ( $\Delta t=30 \mathrm{~min})$ predominates. For larger distances, the contribution of spatial variability becomes significant. In contrast to this, $\sigma_{\mathrm{IWV}}$ is not increasing with $\Delta x$ in the winter season (Fig. 2, blue curve). This is in agreement with the assumption that local convection does not reach the vertical measurement range during the winter season and that the IWV variability is probably dominated by horizontal advection of filamentary structures in the free troposphere from very different source regions. Consequently, the observed variability during winter is due to larger spatial scale processes (compared to local convection in summer), which would explain the absence of an increase with $\Delta x$ in Fig. 2. Note that because IWV is much lower in winter than in summer, the relative variabilities (i.e. if $\sigma_{\mathrm{IWV}}$ were given in per cent) would be larger for the blue curve in Fig. 2. This means that advec- 
tion of filaments (winter) leads to larger relative changes of IWV than local convection in summer. We will discuss this finding in more detail within the context of the variability of the vertical water vapor profile in Sect. 3 . Figure 2 also indicates that $\sigma_{\mathrm{IWV}}$ even shows a trend to lower values for distances above $6 \mathrm{~km}$. We explain this by the fact that measurements with large horizontal mismatch $(\Delta x>6 \mathrm{~km})$ require extraordinarily calm and clear weather conditions, because the FTIR instrument needs a cloudless field of view while the sun position is close to the horizon. While the mean horizontal distance is low during the summer season $(\Delta x<2 \mathrm{~km})$ due to high sun positions and stronger cloud perturbance, it reaches up to almost $10 \mathrm{~km}$ around midwinter. The variability over the entire field of horizontal distances within a certain time interval (e.g. $20 \mathrm{~min}$ ) reaches its maximum of almost $0.6 \mathrm{~mm}$ when the temperature peaks around the end of July. We assume that this is a direct effect of the heat-driven local convection, which can reach altitudes of $4.5 \mathrm{~km}$ at Zugspitze site during the summer season. As expected, the minimum variability of about $0.15 \mathrm{~mm}$ is observed around midwinter, although the mean horizontal mismatch of both instruments is largest at this time of the year. However, as stated above, relative short-term variations are larger during the winter season.

\section{Temporal variability}

For the analysis of temporal variability, we calculated the standard deviation of differences $\sigma_{\text {IWV }}$ between IWV values from both instruments as a function of temporal coincidence. This was repeated for varied spatial matching criteria. When using all IWV values from both instruments without applying any geometrical matching criteria, $\sigma_{\mathrm{IWV}}$ shows a flat minimum around a coincidence interval of $\Delta t=20 \mathrm{~min}$ (red curve in Fig. 3). The minimum is assigned to the fact the most FTIR and lidar measurements were carried out in the morning, because there are still few clouds. As a consequence, most of the pairs with the shortest coincidence intervals are found in the morning when the spatial matching is worst. This slightly increases $\sigma_{\mathrm{IWV}}$ on the very left hand side of the red curve in Fig. 3. Secondly, many pairs with good spatial matching can be found around noon, even for somewhat larger temporal coincidence intervals. This explains the decrease of $\sigma_{\mathrm{IWV}}$ towards the minimum. When considering measurement pairs with an FTIR sun azimuth close to the position of the DIAL instrument $\left(210^{\circ} \pm 6^{\circ}\right)$ only, $\sigma_{\text {IWV }}$ is much smaller in general and has its minimum at the shortest coincidence intervals (green curve in Fig. 3). For time intervals on the minutes scale, we find $\sigma_{\text {IWV }}=0.05 \mathrm{~mm}$, which agrees with the validated (combined) precision of our instruments [4]. The temporal variability of IWV can also be estimated from the standard deviation of differences of measurements recorded by the same instrument within certain time intervals. This was done with data from the FTIR instrument (blue curve in Fig. 3). They begin to deviate increasingly from the green curve beyond 30 minutes and converge towards the red curve for larger time intervals. This corresponds

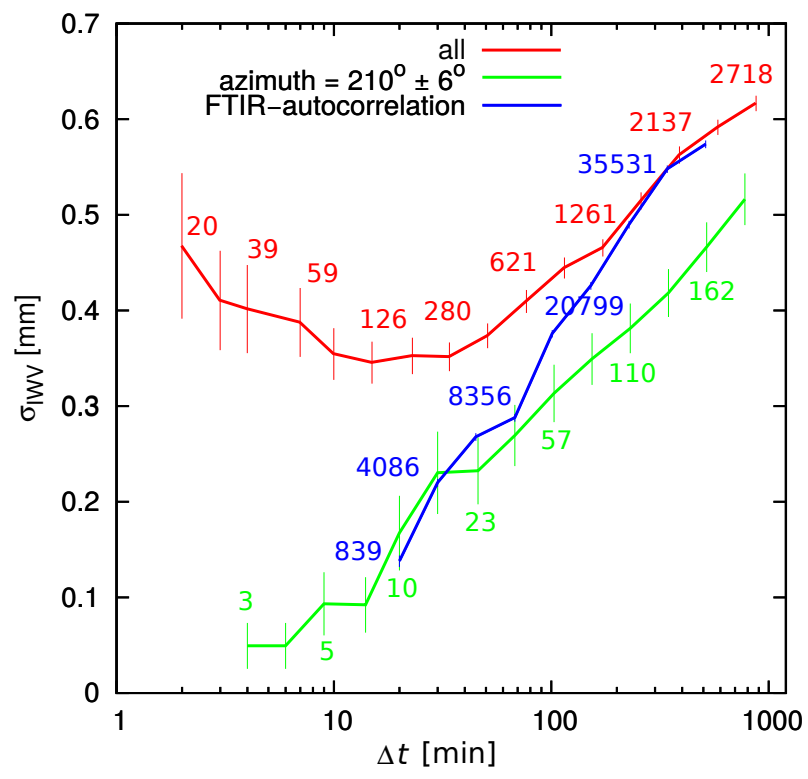

Figure 3: Variability as a function of the length of the time interval. The red curve shows $\sigma_{\mathrm{IWV}}$ from all measurements with no geometrical restrictions as a function of the length of the time interval in which data were taken into account. The green curve only includes measurements recorded in the early afternoon when the volume matching peaks with a sun azimuth of $210^{\circ} \pm 6^{\circ}$. The blue curve only shows $\sigma_{\text {IWV }}$ of IWV values from the FTIR instrument. The quantity of measurement pairs from which $\sigma_{\mathrm{IWV}}$ was calculated is indicated by the numbers near the curves (not for all nodes). The uncertainties $( \pm \sigma)$ are indicated by the error bars (for calculation see text).

to the fact that we observe a superposition of temporal and spatial variability with the solar FTIR due to its sunpointing geometry.

\section{PROFILE VARIABILITY}

The variability of the vertical water vapor distribution on time scales of $\Delta t \leq 5 \mathrm{~h}$ was derived from profiles retrieved from the DIAL measurements alone (Fig. 4). For the lowest layer (i.e. $3-4 \mathrm{~km}$ ), where most of the entire column above Zugspitze site is located, we find equal relative variabilities as for IWV. In contrast to this, the relative variability increases with altitude above $5 \mathrm{~km}$. This can be explained by the increasing wind speed at higher altitudes in the troposphere. The temporal variability of the water vapor density in the free troposphere at a certain altitude primarily features a horizontal variability combined with a horizontal wind velocity at this altitude. Depending on the pathway of the jet stream or the polar vortex, maximum wind velocities of more than $100 \mathrm{~m} / \mathrm{s}$ occur. Considering a time interval of $60 \mathrm{~min}$, this means a mean horizontal spread of about $80 \mathrm{~km}$ around $10 \mathrm{~km}$ altitude with a potential increase to more than $360 \mathrm{~km}$ in the jet stream regime. For different time intervals ( $1 \mathrm{~h}-5 \mathrm{~h})$, the relative variance behaves quite similarly in agreement 


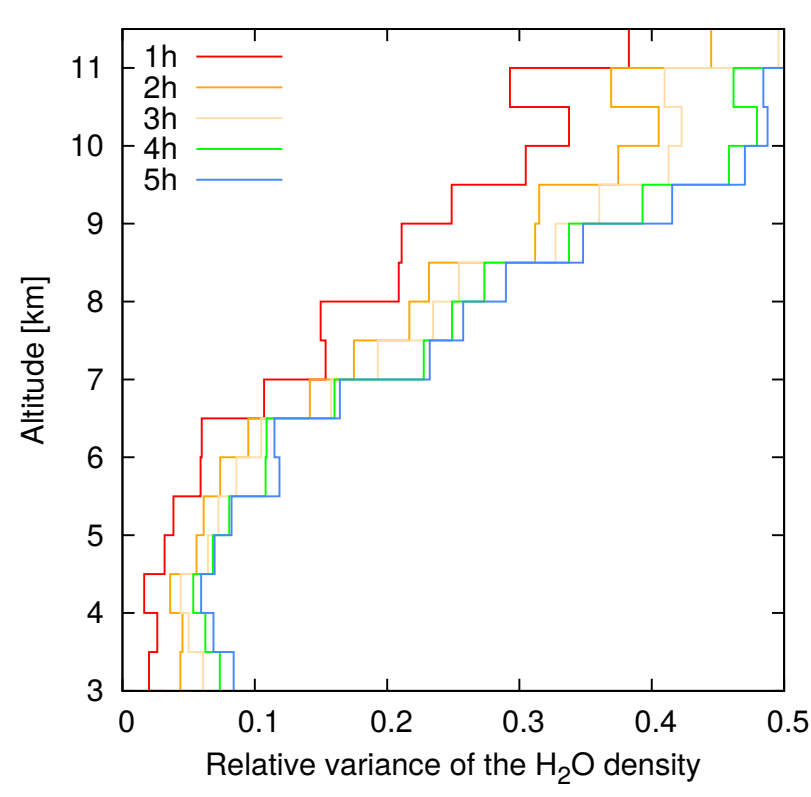

Figure 4: The short-term variability of the vertical water vapor profile is illustrated by the plot of the relative variance as a function of altitude within different time intervals.

with our results of IWV variability analysis, according to which longer time intervals lead to larger variabilities. In comparison to the $1-\mathrm{h}$ profile, we see a more significant maximum at the lower edge at $3 \mathrm{~km}$ and a significant minimum at $4.5 \mathrm{~km}$ for longer time intervals. This enhanced increase between $3 \mathrm{~km}$ and $4 \mathrm{~km}$ is, to our understanding, induced by the diurnally varying upper edge of the boundary layer during the warm season.

The interconnectivity of the short-term variability of water vapor at different altitudes is analyzed using the covariance matrix of the vertical profile variability. A weak coupling between different layers is observed for lower altitudes which is in agreement with the assumption of local convection and turbulence being the dominant sources of variability in the lower part of the examined altitude range. In the upper troposphere, on the other hand, varying air masses are more coherently exchanged within the upper air flow, as a result of which layers of a wider vertical spread are affected.

\section{MECHANISMS DRIVING THE OBSERVED VARIABILITY}

Lidar sounding allows for clearly distinguishing between local convection and long-range transport as sources of variability, though also a superposition of both mechanisms can be observed. From these incidents we learned that the water-vapor density at a certain altitude can vary by a factor of more than 30 within a few hours. Thus, the short-term variability of water vapor induced by longrange transport and the advection of very inhomogeneous layer structures can exceed the impact of local convection by roughly one order of magnitude. This is particu- larly pronounced for stratospheric intrusions that descend from the Arctic to Central Europe. These intrusion layers occasionally become the main source of short-term variability of water vapor in the altitude range between $3 \mathrm{~km}$ and $5 \mathrm{~km}$ [5]. However, such events occur predominantly during the winter season and are accompanied with nonconvective weather conditions. Case studies based on lidar observations will be presented at the ILRC-meeting.

\section{CONCLUSIONS}

Our results for the first time provide a quantitative description of the free tropospheric spatio-temporal variability of water vapor in the free troposphere on the scales of minutes and kilometers (horizontal) for IWV and on the scales of hours and $500 \mathrm{~m}$ (vertical) for profiles. This information can be useful for the parameterization of humidity in atmospheric models as well as for estimating the influence of the atmospheric variability of water vapor on the significance of water vapor measurements performed with a given integration time. In a related sense our results also provide the information necessary for evaluating intercomparison studies of not perfectly colocated or synchronized instruments.

\section{ACKNOWLEDGMENTS}

We thank Hans-Peter Schmid (KIT/IMK-IFU) for his continuous interest in this work and $\mathrm{M}$. Rettinger (KIT/IMK-IFU) for executing the FITR measurements at Mt. Zugspitze. We also acknowledge the assistance by the team of the Schneefernerhaus research station (UFS) and the Bavarian Ministry of Environment and Consumer Protection for funding our work within the ALOMAR cooperation.

\section{REFERENCES}

1. Vogelmann, H., Sussmann, R., Trickl, T., and Reichert, A. Spatiotemporal variability of water vapor investigated using lidar and ftir vertical soundings above the zugspitze. Atmos. Chem. Phys., 15:3135-3148, 2015.

2. Sussmann, R. and Schäfer, K. Infrared spectroscopy of tropospheric trace gases: combined analysis of horizontal and vertical column abundances. Appl. Opt., 36:735-741, 1997.

3. Vogelmann, H. and Trickl, T. Wide Range Sounding of Free Tropospheric Water Vapor with a Differential Absorption Lidar (DIAL) at a High Altitude Station. Appl. Opt., 47(12):2116-2132, 2008.

4. Vogelmann, H., Sussmann, R., Trickl, T., and Borsdorff, T. Intercomparison of atmospheric water vapor soundings from the differential absorption lidar (DIAL) and the solar FTIR system on Mt. Zugspitze. Atmos. Meas. Tech., 4(5):835-841, 2011.

5. Trickl, T., Vogelmann, H., Giehl, H., Scheel, H.-E., Sprenger, M., and Stohl, A. How stratospheric are deep stratospheric intrusions? Atmos. Chem. Phys., 14(18):9941-9961, 2014. 